\title{
INFECÇÃO URINÁRIA PÓS-TRANSPLANTE RENAL E USO DE CATETER URETERAL DUPLO J
}

\author{
Urinary tract infection in kidney transplantation and its association with the use of ureteral stents
}

Cláudia Maria Costa de Oliveira, João Batista Gadelha Cerqueira, Daniela Costa de Oliveira Santos, Márcia Uchoa Mota, Silvana Albuquerque Andrade, Evelyne Santana Girão, Leyla Castelo Branco Marques, Wilson Mendes Barroso, Ailson Gurgel Fernandes,

Paula Castelo Branco Camurça Fernandes, João Evangelista Júnior

\section{RESUMO}

Introdução: $\mathrm{O}$ uso de cateter ureteral duplo J na cirurgia de transplante renal tem sido associado à redução nas complicações urológicas, mas seu impacto na frequência de infecção do trato urinário (ITU) é variável. Objetivos: Determinar a frequência de ITU pós-transplante renal dos patógenos envolvidos, da utilização de cateter ureteral duplo J na população em estudo, bem como avaliar o impacto da utilização de cateter duplo J na frequência de ITU em receptores de Transplante renal. Métodos: Foram incluídos no presente estudo de coorte retrospectivo todos os receptores submetidos a transplante renal no Hospital Universitário Walter Cantídeo no período entre janeiro de 1998 a agosto de 2004, com pelo menos três meses de seguimento pós-transplante. A coleta dos dados foi realizada através de revisão dos prontuários e fichas de acompanhamento ambulatorial desde o transplante até o período final do seguimento. Foi avaliada a frequência de ITU, o tempo pós-transplante do diagnóstico da ITU, os principais patógenos envolvidos, a terapia utilizada para a ITU, a frequência do uso de cateter ureteral duplo J e da ocorrência da complicação de fístula urinária na população em estudo. Resultados: A frequência de ITU na população em estudo foi de $47 \%$. Os principais patógenos identificados foram Klebsiella pneumoniae, Escherichia coli e Pseudomonas aeruginosa. Um cateter ureteral duplo J foi utilizado em $69 \%$ dos pacientes, com permanência média de 20 dias. O uso de cateter ureteral duplo $\mathrm{J}$ na cirurgia do transplante não teve impacto na frequência de fístula urinária, mas esteve associado a uma chance 2,98 vezes maior de desenvolvimento de ITU, quando comparado ao grupo que não utilizou duplo J. Conclusões: A incidência de ITU na população em estudo foi de $47 \%$, Um cateter ureteral duplo J foi utilizado em $69 \%$ dos pacientes e não apresentou impacto na frequência de fístula urinária, mas esteve associado a uma chance 2,98 vezes maior de desenvolver ITU.

Descritores: Infecções Urinárias, Transplante de Rim, Infecções Relacionadas a Cateter

Instituição:

Transplante Renal do Hospital Universitário Walter Cantídeo - Universidade Federal do Ceará (UFC) - Fortaleza/CE, Brasil.

\section{Correspondência:}

Claudia Maria Costa de Oliveira

Nefrologia, Universidade Federal do Ceará - Brasil

Rua Professor Jacinto Botelho, 500 / 500 - CEP 60.810-050 - Fortaleza/CE

Tel.: (85) 3241-2036 Fax: (85) 3366-8611

E-mail: claudiadrl@gmail.com

Recebido em: 06/12/2011

Aceito em: 15/01/2012

\section{INTRODUÇÃO}

A infecção do trato urinário (ITU) é a infecção bacteriana mais comum em transplante (Tx) renal, com incidência variável entre 6-86\% dos receptores (também descrita como 35-69\%) e contribui com 40 a $50 \%$ de todas as complicações infecciosas nesse grupo de pacientes..$^{1-5} \mathrm{~A}$ incidência variável pode ser atribuída a diferenças na definição de ITU, método de coleta da urina e uso ou não de profilaxia antibiótica no pós-operatório. ${ }^{6}$

A ITU é mais comum no pós-operatório precoce (três a seis meses pós-Tx) e tem um efeito direto na morbimortalidade do receptor. As complicações mais comuns relacionadas à ITU pós-Tx renal são: septicemia, ocorrendo em 40 a $60 \%$ dos transplantados; reinfecção, que geralmente ocorre por um patógeno diferente daquele da infecção precedente; rejeição crônica, cujo risco aumenta após três anos de transplantee e infecção por citomegalovírus, que é muito frequente após episódio de ITU febril..$^{7-9}$

As manifestações clínicas mais comuns apresentadas por pacientes transplantados renais com ITU são bacteriúria, piúria, cistite aguda, pielonefrite e septicemia. ${ }^{10}$ 
O risco de ITU em pacientes transplantados é determinado principalmente por dois fatores: a intensidade da exposição a patógenos, que difere na comunidade e no hospital, e a natureza da terapia imunossupressora, essa última definida por dose e duração da imunossupressão. ${ }^{11}$ Outros fatores relatados na literatura que podem estar associados a maior risco de desenvolvimento de ITU pós-Tx renal são: idade avançada, sexo feminino, ${ }^{12}$ receptor de doador falecido, ${ }^{13}$ raça negra, duração da cateterização vesical,${ }^{14}$ anormalidades funcionais ou neurogênicas, bacteriúria pré-transplante, doença renal crônica primária com obstrução, pielonefrite crônica, doença renal policística, história de diabetes e episódio de rejeição aguda nos primeiros seis meses. ${ }^{1,15}$

$\mathrm{O}$ uso de cateter ureteral duplo $\mathrm{J}$ na cirurgia do transplante renal tem sido associado à redução das complicações urológicas, mas o seu impacto na frequência de ITU é variável. ${ }^{16}$

Foram objetivos do presente estudo, determinar a frequência de ITU pós-Tx renal, dos patógenos envolvidos, da utilização de cateter ureteral duplo J na população em estudo, bem como avaliar o impacto da utilização de cateter duplo J na frequência de ITU em receptores de Tx renal.

\section{MATERIAL E MÉTODOS}

Foram incluídos neste estudo de coorte retrospectivo todos os receptores submetidos a transplante renal no Hospital Universitário Walter Cantídeo - HUWC, no período entre janeiro de 1998 e agosto de 2004, com pelo menos três meses de seguimento pós-transplante.

A coleta dos dados foi realizada através de revisão dos prontuários e fichas de acompanhamento ambulatorial desde o Tx até o período final do seguimento.

Infecção do trato urinário foi definida como episódios com urinocultura (UC) positiva, ainda que assintomáticos e episódios com sumário de urina infecciosa, associados à febre e/ou disúria, em que não tenha sido possível efetuar coleta de UC antes da antibioticoterapia.

Foi avaliada a frequência de ITU, o tempo pós-transplante do diagnóstico da ITU, os principais patógenos envolvidos, a terapia utilizada para a ITU, a frequência do uso de cateter ureteral duplo $\mathrm{J}$ e da ocorrência da complicação de fístula urinária na população em estudo.

O projeto foi submetido à avaliação e aprovação do Comitê de Ética do HUWC e foram respeitados todos os princípios éticos que regem a lei 196/96 do Conselho Nacional de Saúde - CSN/ Ministério da Saúde - MS, que regulamenta a pesquisa em seres humanos.

Os resultados foram expressos em média \pm desvio padrão. As variáveis com distribuição normal foram comparadas pelo teste $\mathrm{t}$ de Student e naquelas com distribuição não normal, foi utilizado o teste Mann-Whitnney.

O teste do qui-quadrado foi utilizado para testar a diferença entre as variáveis demográficas, clínicas e laboratoriais nos grupos com e sem ITU. Um valor de $\mathrm{p}$ inferior a $5 \%$ foi considerado estatisticamente significante. A análise estatística foi realizada utilizando-se o programa estatístico SPSS 14.0.

\section{RESULTADOS}

Foram realizados 210 transplantes renais no período em estudo, sendo $134(63,8 \%)$ com doador falecido e $76(36,2 \%)$ com doador vivo. A idade média dos receptores foi de 36,9 anos (variando de 12-65 anos), sendo 122 pacientes (58\%) do sexo masculino. Durante o período de seguimento, foram diagnosticados 99 pacientes $(47 \%)$ com pelo menos um episódio de ITU pós-Tx renal. O critério utilizado para o diagnóstico de ITU foi a urinocultura, em 93,3\% dos casos. Entre os pacientes com ITU, 58\% apresentaram apenas um episódio de ITU e 42\% apresentaram mais de um episódio de ITU (Figura 1) O tempo médio de aquisição do primeiro episódio de ITU foi de 17 dias (variando de dois a 199 dias), e para os episódios ocorridos nos primeiros 30 dias pós-TX renal, o tempo médio de ocorrência da ITU foi de nove dias. Não houve diferença na frequência de ITU de acordo com o sexo (masculino: 48,3\% e feminino: $45,4 \% ; \mathrm{p}=0,17$ ) e o tipo de doador (vivo: $40,7 \%$ e falecido: $50,7 \% ; \mathrm{p}=0,16)$.

O tempo para o diagnóstico do primeiro episódio de ITU pós-Tx renal foi inferior a 30 dias em $87 \%$ dos casos, entre 30-90 dias em 10,5\% e após 90 dias em 2,3\% dos pacientes (Figura 2).

Figura 1. Número de episódios de infecção urinária no período em estudo. HUWC-Fortaleza/CE

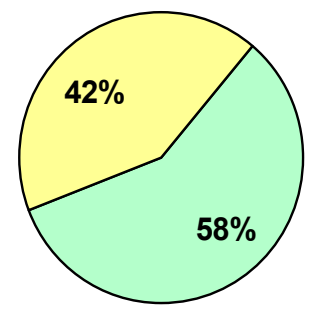

\section{$\square 1$ episódio $\square>1$ episódio}

O tempo para o diagnóstico do primeiro episódio de ITU pós-Tx renal foi inferior a 30 dias em $87 \%$ dos casos, entre $30-90$ dias em $10,5 \%$ e após 90 dias em 2,3\% dos pacientes (Figura 2).

Figura 2. Tempo pós-transplante renal do diagnóstico do primeiro episódio de infecção do trato urinário na população em estudo. HUWC, Fortaleza-CE

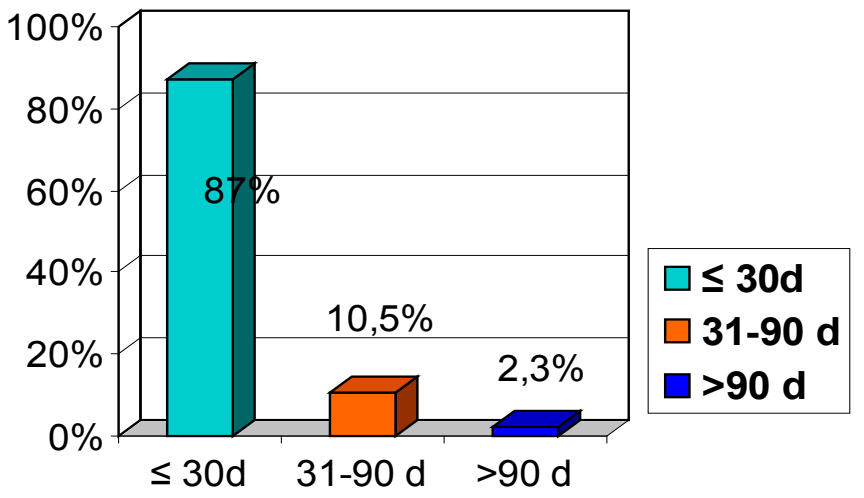

$\mathrm{Na}$ Tabela 1, estão apresentados os principais patógenos isolados nas urinoculturas realizadas, sendo a Klebsiella pneumoniae a bactéria mais prevalente.

Foram ainda isolados em menor percentual de casos, os seguintes 
agentes etiológicos: Candida sp, Citrobacter, Morganella, Serratia, S. aureus, S. viridans.

Os episódios de ITU foram tratados com os seguintes antibióticos: imipenem em $39 \%$ dos casos, quinolonas em $27 \%$, cefalosporinas de $3^{\mathrm{a}}$ geração em $23,5 \%$, seguidos por cefepime em $3 \%$, cefoxitina em $2,4 \%$ e vancomicina em $2,4 \%$.

$\mathrm{O}$ cateter ureteral duplo $\mathrm{J}$ foi colocado no momento do transplante em 145 pacientes (69\% dos casos) e o tempo médio de permanência do cateter duplo $\mathrm{J}$ foi de 20,7 dias (variando de 3-90 dias).

Tabela 1. Frequência dos patógenos isolados nas urinoculturas realizadas na população em estudo. HUWC, Fortaleza-CE.

\begin{tabular}{c|c}
\hline Patógeno & Percentual \\
\hline K. pneumoniae & $49,1 \%$ \\
E. coli & $22,3 \%$ \\
P. aeruginosa & $10,8 \%$ \\
Enterobacter cloaceae & $6,5 \%$ \\
Enterococus faecalis & $2,8 \%$ \\
Outros & $8,5 \%$ \\
\hline
\end{tabular}

Os receptores que receberam um enxerto de doador falecido apresentaram uma chance 2,45 vezes maior de usar cateter duplo $\mathrm{J}$, quando comparados aos receptores de doador vivo $(\mathrm{OR}=2,45$ [1,28-4,68] 95\% IC; $p=0,005)$ e os pacientes do sexo masculino tiveram uma chance 0,45 vezes menor de usar duplo J, quando comparados aos do sexo feminino $(\mathrm{OR}=0,45[0,23-0,89] 95 \% \mathrm{IC}$; $\mathrm{p}=0,01)$.

Infecção urinária pós-transplante renal foi diagnosticada em $56 \%$ dos pacientes com duplo $\mathrm{J}$ e $28,3 \%$ dos pacientes que não fizeram uso de duplo J, representando uma chance 2,98 vezes maior de ITU com o uso do cateter ureteral duplo J $(\mathrm{OR}=2,98[1,52-5,86] 95 \%$ IC $; \mathrm{p}<0,005$ ) (Figura 3).

A frequência de fístula urinária no grupo que usou cateter duplo $\mathrm{J}$ foi de $5,5 \%$, e de $0 \%$ no grupo que não utilizou duplo $\mathrm{J}(\mathrm{p}=\mathrm{NS})$.

Figura 3. Frequência de infecção do trato urniário pós-transplante segundo o uso ou não de cateter ureteral duplo J na população em estudo. HUWC, Fortaleza-CE.

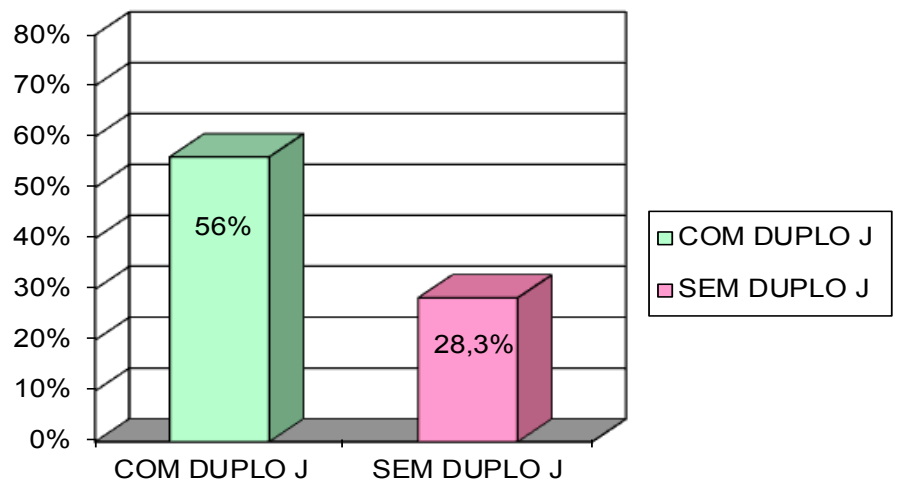

\section{DISCUSSÃO}

A prevalência de ITU pós-Tx renal no presente estudo foi de $47 \%$. $\mathrm{Na}$ literatura, a prevalência de ITU pós-Tx renal varia de 35 a $80 \%$, caindo progressivamente durante os primeiros anos após o transplante, variando de $35 \%$ no primeiro ano, até praticamente desaparecer após cinco anos. ${ }^{17,18}$

Segundo Ferreira e Heilberg, as infecções do trato urinário ocorrem, na maioria das vezes, nos três primeiros meses após o Tx renal, ${ }^{18}$ tendo sido o mesmo observado no presente estudo, onde $97,5 \%$ dos episódios foram diagnosticados nos três primeiros meses pós-Tx renal. O tempo médio de aquisição da ITU pós-Tx renal tem sido descrito entre quatro a sete dias ${ }^{19}$ e o risco maior está relacionado à imunossupressão, vulnerabilidade do enxerto após manipulação cirúrgica, cateter vesical, entre outros. O tempo de internamento tem sido descrito como de 27 dias para pacientes sem ITU e 36 dias para aqueles com ITU pós-Tx. ${ }^{20}$ No presente estudo, o tempo de aquisição do primeiro episódio de ITU foi de 17 dias e o tempo de internamento foi de $31,8 \pm 21,2$ dias para os pacientes com ITU e 24,4 $\pm 21,3$ dias para os que não evoluíram com ITU. Os mesmos autores relataram uma maior frequência de ITU pós-Tx renal em mulheres do que em homens, mas no presente estudo não houve diferença na frequência de ITU segundo o gênero.

Diferentemente do que relata a literatura, ${ }^{12,13,20}$ os autores não encontraram uma maior prevalência de ITU no sexo feminino (masculino: 48,3\% e feminino: $45,4 \% ; \mathrm{p}=0,17$ ) ou em receptor de doador falecido (vivo: $40,7 \%$ e falecido: $50,7 \%$; $=0,16$ ). É provável que a utilização de órgãos de doadores vivos esteja associada a menor prevalência de ITU devido ao menor tempo de isquemia fria, menor grau de injúria isquemia-reperfusão e menor taxa de função retardada do enxerto. ${ }^{21}$

Na literatura, há um amplo espectro de patógenos causando ITU pósTx renal, e as bactérias que causam ITU em imunossuprimidos são semelhantes àquelas que causam ITU em não imunossuprimidos. Na população em geral, E. coli é responsável por $80 \%$ dos episódios de ITU. ${ }^{22}$ No Tx renal, E. coli tem sido identificada em $29-60 \%$ dos casos. ${ }^{23,24}$ Vários autores ainda descrevem a E. coli como o principal patógeno das ITUs pós-Tx renal. ${ }^{25-27}$

Proteus mirabilis e Klebsiella pneumoniae têm sido identificados em até $30 \%$ das urinoculturas e cocos gram positivos em $20 \%$ das culturas [10]. Chuang et al., estudando 213 episódios de ITU em 500 receptores, ao longo de 42 meses pós-Tx, encontraram como principais patógenos nas urinoculturas: E. coli $(29 \%)$, Enterococcus (24\%), Staphylococcus (12\%) e Klebsiella (10\%) [13]. Por outro lado, em estudo da Turquia, E. coli foi isolada em $61,3 \%$ dos episódios de ITU pós-Tx. ${ }^{28}$

No presente estudo, detectamos maior prevalência de ITU por K. pneumoniae $(49,1 \%)$, seguido por E. coli $(22,3 \%)$ e P. aeruginosa $(10,8 \%)$.

A cateterização ureteral com duplo $\mathrm{J}$ no transplante renal tem como objetivo diminuir ou eliminar a ocorrência de complicações urológicas. ${ }^{16,29} \mathrm{O}$ cateter duplo J constitui-se um corpo estranho, que pode predispor a ocorrência de infecções e impactar negativamente a função do enxerto a longo. ${ }^{30} \mathrm{O}$ presente estudo mostrou maior incidência de infecção urinária em pacientes submetidos à colocação de duplo J $(56 \%$ x $23,8 \%)$ e não houve relação entre complicação urológica com a presença ou não do cateter.

$\mathrm{Na}$ literatura, os resultados são conflitantes. Em meta-análise realizada, Mangus et al. mostraram que a utilização do cateter duplo $\mathrm{J}$ poderia diminuir a taxa de complicações urológicas de $33 \%$ para $6 \% .{ }^{31}$ Estudos retrospectivos apontam diminuição das complicações urológicas, ${ }^{32}$ enquanto os ensaios prospectivos randomizados produzem resultados controversos. ${ }^{33}$ Benoit et al. 
compararam prospectivamente a frequência de complicações urológicas (fístulas, obstruções e ITU) em 194 receptores de Tx renal (97 com cateter ureteral duplo J e 97 sem duplo J). ${ }^{34}$ No grupo com duplo J, um paciente apresentou fístula urinária e 35 evoluíram com ITU. No grupo sem cateter duplo J, seis pacientes complicaram com fístula urinária, quatro com obstrução urinária e 32 com ITU. A sobrevida dos pacientes e enxertos e a função renal, após um ano de Tx, foi semelhante nos dois grupos e os autores concluíram que a inserção do cateter duplo J diminuiu a taxa de fístula e obstrução urinária em Tx renal.

A presença de um número maior de pacientes com fístula urinária e cateter duplo J no presente estudo é explicada pela inclusão de pacientes que apresentaram complicações urológicas no póstransplante e foram tratados com a colocação de cateter duplo J. Apesar da controvérsia, todos os estudos apontam que, tão ou mais importante que a colocação do duplo J, a diminuição das complicações urológicas pode ser conseguida com a preservação da vascularização ureteral, técnica de implante extravesical e diminuição da dose de esteróides nos esquemas imunossupressores. ${ }^{31,33}$ Dois trabalhos da literatura apontam taxas de infecção urinária com a presença do duplo $\mathrm{J}$ semelhantes ao observado neste trabalho. Tavakoli et al. reportaram taxas de infecção em torno de $33 \%{ }^{16}$ e Ranganathan et al. de $71 \%{ }^{30}$

No presente trabalho, não foi estudada a relação entre o tempo de permanência do cateter e a incidência de infecção urinária, embora o tempo médio de permanência do duplo $\mathrm{J}$ tenha sido de 20 dias. Em estudo realizado por Coskun et al. ${ }^{35}$ o tempo crítico para o processo de cicatrização das anastomoses urológicas situa-se aproximadamente em 14 dias. ${ }^{35} \mathrm{O}$ autor avaliou os pacientes que retiraram o duplo $\mathrm{J}$ em um tempo inferior a 14 dias contra aqueles que foram submetidos à retirada do cateter em tempo superior a 20 dias. A retirada do cateter em um tempo inferior a 14 dias fez com que a taxa de infecção urinária diminuísse de $35 \%$ para $2 \%{ }^{35} \mathrm{O}$ tempo médio de permanência do duplo $\mathrm{J}$ nos pacientes do presente estudo, 20 dias, pode explicar a taxa de infecção urinária aqui relatada.

Glazier et al. demonstraram que o uso de cateter ureteral duplo J é seguro, mas associado a uma taxa de ITU de $22,6 \% .{ }^{36}$ Para reduzir as taxas de ITU, eles recomendam a remoção do cateter ureteral com 14 dias e mais cedo, se possível, principalmente em diabéticos que receberam um enxerto de doador falecido.

Alguns centros de transplante podem ter uma prevalência relativamente alta de complicações urológicas e nesses casos os receptores podem ter benefícios com o uso de stent ureteral duplo J através de uma redução significativa na taxa de fístula urinária ou obstrução ureteral. ${ }^{16,37}$ Contudo, uma elevação significativa na taxa de ITU pode ser observada quando esses stents permanecem por mais de 30 dias pós-Tx, sendo sugerido que eles sejam removidos antes de quatro semanas.

\section{CONCLUSÃO}

A frequência de ITU na população em estudo foi de $47 \%$. Os principais patógenos identificados foram Klebsiella pneumoniae, Escherichia coli e Pseudomonas aeruginosa.

Um cateter ureteral duplo J foi utilizado em $69 \%$ dos pacientes, com permanência média de 20 dias. O uso de cateter ureteral duplo J na cirurgia do Tx não teve impacto na frequência de fístula urinária, mas esteve associado a uma chance 2,98 vezes maior de desenvolvimento de ITU, quando comparado ao grupo que não utilizou duplo $\mathrm{J}$.

\section{ABSTRACT}

Introduction: The use of double-J ureteral stent in renal transplantation has been associated to the reduction in the urologic complications, but its impact on the frequency of UTI is variable. Purpose: This study was designed to determine the frequency of UTI after renal Tx, the pathogen involved, frequency of use of double-J ureteral stent in the studied population and to assess the impact of the use of double-J stent on the frequency of UTI in kidney transplanted recipients. Methods: We included in this retrospective cohort study all kidney transplant performed at Walter Cantídeo University Hospital between January 1998 and August 2004 with at least 3 months of post-transplant follow-up. Data collection was performed by reviewing medical records and ambulatory charts from the transplant up to the end of follow-up period. We assessed the frequency of UTI, the post-transplant time of UTI diagnosis, the main pathogens involved, used therapy, frequency of use of double-J urethral stent and occurrence of urinary fistulae in the studied population. Results: The frequency of UTI was $47 \%$. The main pathogens identified were Klebsiella pneumoniae, Escherichia coli and Pseudomonas aeruginosa. A double-J ureteral stent was used in $69 \%$ of patients with average stay of 20 days. The use of double-J ureteral stent in the Tx surgery had no impact on the frequency of urinary fistula, but it was associated to a 2,98 times higher chance to develop UTI compared to the group which did not use double J. Conclusions: The UTI incidence in the studied population was $47 \%$. A double-J ureteral stent was used in $69 \%$ of patients with no impact on the frequency of urinary fistula, but was associated to a 2,98 times higher chance to develop UTI.

Keywords: Urinary Tract Infection; Kidney Transplantation; Catheter-Related Infections 


\section{REFERÊNCIAS:}

1.Dharnidharka VR, Agodoa LY, Abbott KC. Effects of urinary tract infection on outcomes after renal transplantation in children. Clin J Am Soc Nephrol. 2007;2(1):100-6.

2. Saemann M, Horl WH. Urinary tract infection in renal transplant recipients. Eur J Clin Invest. 2008;38(Suppl 2):58-65.

3. Rubin RH. Infectious disease complications of renal transplantation. Kidney Int. 1993;44(1):221-36.

4. Karakayali H, Emiroglu R, Arslan G et al. Major infectious complications after kidney transplantation. Transplant Proc. 2001;33(1-2):1816-7.

5. Giral M, Pascuariello G, Karam G et al. Acute graft pyelonephritis and longterm kidney allograft outcome. Kidney Int. 2002;61(5):1880-6.

6. Schmaldienst S, Horl WH. Bacterial Infections after renal transplantation. Contrib Nephrol. 1998;124:18-33.

7. Muller V, Becker G, Delfs $M$ et al. Do urinary tract infections trigger chronic kidney transplant rejection in man? J Urology 1998;159(6):182629.

8. Abbott KC. Oliver JD 3rd, Hypolite I et al. Hospitalizations for bacterial septicemia after renal transplantation in the United States. Am J Nephrol. 2001;21(2):120-7

9. Audard V, Amor M, Desvaux D et al. Acute graft pyelonephritis: a potential cause of acute rejection in renal transplant. Transplantation. 2005;80(8):1228-30.

10. Schmaldienst S, Dittrich E, Horl WH. Urinary tract infections after renal transplantation. Curr Opin Urol. 2002;12(2):125-30.

11. Fishman JA, Rubin RH. Infection in organ-transplant recipients. New Engl J Med. 1998;338(24):1741-51.

12. Pelle G, Vimont S, Levy PP et al. Acute pyelonephritis represents a risk factor impairing long-term kidney graft function. Am J Transplant. 2007;7(4):899-907.

13. Chuang P, Parikh CR, Langone A. Urinary tract infections after renal transplantation: a retrospective review at two US transplant centers. Clin Transplant. 2005;19(2):230-5.

14. Hoy WE, Kissel SM, Freeman RB, Sterling WA Jr. Altered patterns of posttransplant urinary tract infections associated with perioperative antibiotics and curtailed catheterization. Am J Kidney Dis.1985;6(4):212-16.

15. Rice JC, Safdar N. Urinary tract infections in solid organ transplant recipients. Am J Transplant. 2009;9(4):267-72.

16. Tavakoli A, Surange RS, Pearson RC et al. Impact of stents on urological complications and health care expenditure in renal transplant recipients: Results of a prospective, randomized clinical trial. J Urol. 2007;177(6):2260-4.

17. Sqalli TH, Laboudi A, Arrayhani M et al. Urinary tract infections in renal allograft recipients from living related donors. Saudi J Kidney Dis Transpl. 2008;19(4):551-3.

18. Ferreira AC, Heilberg IP. Infecção do trato urinário no pós-transplante em crianças. J Bras Nefrol. 2001;23(1):18-24.

19. Midtvedt K, Hartmann A, Midtvedt T, Brekke IB. Routine perioperative antibiotic prophylaxis in renal transplantation. Nephrol Dial Transplant. 1998;13(7):1637-41
20. Kentouni-Noly JC, Cloix P, Martin X et al. Analysis of nosocomial infections in renal transplant and pancreas transplant recipients. Transplant Proc. 1994;26:284.

21. Alangaden GJ, Thyagarajan R, Gruber SA et al. Infectious complications after kidney transplantation: current epidemiology and associated risk factors. Clin Transplant. 2006;20(4):401-9.

22. Foxman B, Brown P. Epidemiology of urinary tract infections: transmission and risk factors, incidence, and costs. Infect Dis Clin North Am. 2003;17(2):227-41.

23. Prát $\mathrm{V}$, Horcicková $\mathrm{M}$, Matousovic $\mathrm{K}$ et al. Urinary tract infection in renal transplant recipients. Infection 1985;13(5):207-10.

24. Takai K, Tollemar J, Wilczek HE, Groth CG. Urinary tract infections following renal transplantation. Clin Transplant. 1998;12(1):19-23.

25. Rice JC, Safdar N and the AST Infectious Diseases Community of Practice. Urinary Tract Infections in Solid Organ Transplant Recipients. Am J Transplant. 2009;9(Suppl 4):S267-S272.

26. Alexopoulos E, Memmos D, Sakellariou G et al. Urinary tract infections after renal transplantation. Drugs Exp Clin Res. 1985;11(2):101-5.

27. Memikoglu KO, Keven K, Sengul S et al. Urinary tract infections following renal transplantation: A single center experience. Transplant Proc. 2007;39(10):3131-4.

28. Senger SS, Arslan H, Azap OK et al. Urinary tract infections in renal transplant recipients. Transplant Proc. 2007;39(4):1016-7.

29. Georgiev P, Boni, C, Dahm F et al. Routine stenting reduces urological complications as compared with stent "on demand" in adult kidney transplantation. Urology 2007;70(5):893-7.

30. Ranganathan $M$, Akbar M, Ilham MA et al. Infective complications associated with ureteral stents in renal transplant recipients. Transplant Proc. 2009;41(1):162-4.

31. Mangus RS, Haag BW. Stented versus non stented extravesical ureteroneocystostomy in renal transplantation: a meta-analysis. Am J Transplant. 2004;4 (11):1889-96.

32. Moray G, Yagmurdur MC, Sevmis S. et al. Effect of routine insertion of a double $\mathrm{j}$ stent after living related renal transplantation. Transplant Proc. 2005;37(2):1052-3.

33. Dominguez J, Clase $\mathrm{CM}$, Mahalati $\mathrm{K}$ et al. Is routine ureteric stenting needed in kidney transplantation? A randomized trial. Transplantation 2000;70(4):597-601.

34. Benoit, G. Blanchet P, Eschwege P et al. Insertion of a double pigtail ureteral stent for the prevention of urological complications in renal transplantation: a prospective randomized study. J Urol. 1996,156(3):881-4.

35. Coskun AK, Harlak A, Ozer T et al. Is removal of the stent at the end of two weeks helpful to reduce infectous or urological complications after renal transplantation? Transplant Proc. 2011;43(3):813-5.

36. Glazier DB, Jacobs MG, Lyman NW et al. Urinary tract infection associated with ureteral stents in renal transplantation. Can J Urol. 1998,5(1):462-6.

37. Pleass HC, Clark KR, Rigg KM al. Urologic complications after renal transplantation: a prospective randomized trial comparing different techniques of ureteric anastomosis and the use of prophylactic ureteric stents. Transplant Proc. 1995;27(1):1091-2. 